\title{
Obstrucción intestinal por Ascaris lumbricoides en un adulto mayor
}

\author{
Alfredo Chiappe, Kovy Arteaga, Cristhian Resurrección, Marcos Navincopa y Eduardo Ticona
}

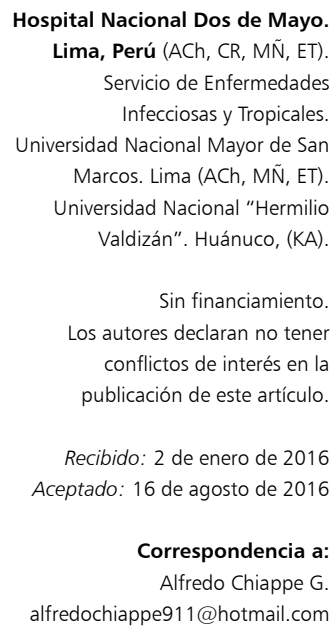

Hospital Nacional Dos de Mayo. Lima, Perú (ACh, CR, MÑ ET). Servicio de Enfermedades Infecciosas y Tropicales. Universidad Nacional Mayor de San Marcos. Lima (ACh, MÑ, ET). Universidad Nacional "Hermilio Valdizán". Huánuco, (KA)

Sin financiamiento Los autores declaran no tener conflictos de interés en la publicación de este artículo.

Recibido: 2 de enero de 2016 Aceptado: 16 de agosto de 2016

Correspondencia a: Alfredo Chiappe G. alfredochiappe911@hotmail.com

\section{Intestinal obstruction due to Ascaris lumbricoides infection in a geriatric patient}

Ascaris lumbricoides is considered the largest intestinal nematode with a higher incidence in the childhood, representing a truly medical and public health problem, principally in undeveloped countries. We present the case of an 83 year old man, born and coming from the amazon region, without any relevant previous history of disease, admitted in the emergency department of our hospital for presenting intestinal obstruction and also presumptive biliary obstruction due to multiple balls of parasites, requiring immediate surgical intervention. We emphasize the need of consider this etiologic possibility in the differential diagnosis, that in this particular case, wasn't suspected in the first place.

Key words: Ascaris lumbricoides, ascariasis, intestinal obstruction, biliar obstruction, exploratory laparotomy.

Palabras clave: Ascaris lumbricoides, ascariasis, obstrucción intestinal, obstrucción biliar, laparotomía exploratoria.

\section{Introducción}

L a parasitosis más frecuente y cosmopolita de todas las helmintiasis humanas es la ocasionada por Ascaris lumbricoides. Se estima que, aproximadamente un cuarto de la población mundial está infectada y cerca de 60.000 personas fallecen anualmente a consecuencia de ello ${ }^{1,2}$. La ascariasis presenta una mayor prevalencia en niños de países tropicales y subtropicales, especialmente en las regiones donde abunda la pobreza, el hacinamiento y la mala sanidad ambiental; ocasionando un retraso en el desarrollo físico e intelectual de los $\operatorname{mismos}^{3,4}$. En el Perú, las regiones de mayor incidencia y prevalencia se presentan en la selva, específicamente en el departamento de Loreto, donde más de $50 \%$ de la población general está afectada ${ }^{5,6}$.

El ser humano se infecta al ingerir los huevos larvados del parásito. Posteriormente, tras la liberación de las larvas en el yeyuno, penetrar la mucosa intestinal y realizar el ciclo de Loos, se establecen definitivamente como ejemplares adultos en el intestino delgado. Este proceso tarda dos a tres meses en completarse. La hembra adulta mide, en promedio, 20 a $49 \mathrm{~cm}$ de longitud y se calcula que puede llegar a producir hasta 200.000 huevos diarios; motivo por el cual, hasta las infecciones de menor cuantía son fácilmente detectadas a través del examen directo de deposiciones $^{7,8}$.

La mayoría de los pacientes son asintomáticos. El diagnóstico se realiza por la combinación de exámenes de sangre, de deposiciones y eventualmente, por los hallazgos radiológicos ${ }^{9}$. Así mismo, las complicaciones suelen ser debidas a la gran cantidad de parásitos adultos en el tubo digestivo, producto de la ingesta de una gran cantidad de huevos larvados; dando lugar a cuadros de obstrucción intestinal, complicación descrita, en conjunto con la obstrucción de la vía biliar, como la más común ${ }^{10,11}$.

En la gran mayoría de los pacientes, el tratamiento es médico, basándose en la utilización del pamoato de pirantel, benzimidazoles y la piperazina, siendo esta última de elección para los casos de obstrucción intestinal o biliar, debido a la parálisis flácida que desencadena en el parásito, facilitando su eliminación. Sin embargo, en ocasiones la resolución con el tratamiento médico no se logra, optando por el manejo quirúrgico de urgencia.

\section{Caso clínico}

Varón de 83 años de edad, procedente del departamento de San Martín (Amazonía del Perú), agricultor. Tenía como antecedente una anemia crónica y un episodio de malaria, hacía dos años. Sin otros antecedentes médicos de relevancia.

El cuadro clínico se inició dos meses previos al ingreso, con dolor cólico en mesogastrio, asociado a falta de apetito, náuseas y vómitos. Finalmente consultó en Servicio de Urgencias por intensificación del dolor abdominal, el que se extendía a todo el abdomen, asociado a vómitos incoercibles, con ausencia de eliminación de gases y deposiciones.

Al examen físico se encontraba quejumbroso, taquicárdico y con ictericia de escleras. La auscultación 
reveló ruidos cardíacos arrítmicos, de intensidad normal. En el examen abdominal los ruidos hidroaéreos estaban disminuidos, la pared abdominal se encontraba blanda, depresible y dolorosa en forma difusa. No se evidenció visceromegalia. El resto del examen físico fue considerado no contributorio.

Ante la sospecha de una posible obstrucción intestinal, se solicitó una radiografía de abdomen simple de pie que mostró dilatación de asas intestinales con presencia de múltiples niveles hidroaéreos (Figura 1).

Entre los exámenes de laboratorio destacaba un hemograma con hemoglobina $15,3 \mathrm{~g} / \mathrm{dL}$, hematocrito $44 \%$, 11.570 leucocitos $/ \mathrm{mm}^{3}$, segmentados $81 \%$, sin baciliformes ni eosinófilos, PCR de $386 \mathrm{mg} / \mathrm{L}$, hiperbilirrubinemia directa (bilirrubina total $3,3 \mathrm{mg} / \mathrm{dL}$ y bilirrubina directa 2,7 mg/dL), elevación de GGT $181 \mathrm{U} / \mathrm{L}$ (GOT y GPT normales), hipoalbuminemia de 1,4 g/dL, y elevación de los niveles de urea: $61 \mathrm{mg} / \mathrm{dL}$ y creatinina: $1,5 \mathrm{mg} / \mathrm{dL}$. Las serologías para VIH, HTLV 1-2 y VHC fueron negativas. El HBsAg y RPR también fueron negativos.

Se realizó una laparotomía exploratoria de urgencia por una probable obstrucción intestinal mecánica. Los hallazgos intraoperatorios mostraron una dilatación de asas del intestino delgado que demarcaban en su superficie formas lineares sugerentes de ejemplares de $A$. lumbricoides. En ese momento se decidió el manejo médico, procediendo al cierre de la pared abdominal.

La evolución clínica posquirúrgica fue desfavorable. El paciente evolucionó hacia un shock hipovolémico, observándose la salida espontánea y masiva de gusanos adultos a través de la cavidad oral y fosas nasales. Se decidió una re-intervención quirúrgica de urgencia, en que se evidenció una gran dilatación de asas intestinales, con abundante cantidad de ejemplares adultos, con múltiples zonas de aglomeración en forma de ovillos, que obstruían la totalidad del lumen intestinal (Figura 2). Se realizó una enterotomía a nivel del íleon, a $40 \mathrm{~cm}$ de la válvula ileocecal, con extracción de 20 gusanos. Se ordeñaron las asas intestinales, desde el ángulo de Treitz hasta la enterotomía, eliminando abundante cantidad de deposiciones con parásitos. En la cavidad abdominal se apreció líquido seroso claro. El apéndice cecal y vesícula biliar eran de características normales.

Luego de la primera cirugía, se inició tratamiento antiparasitario con albendazol. Sin embargo, la evolución clínica fue insatisfactoria, requiriendo luego de la segunda intervención quirúrgica, soporte ventilatorio y manejo en la Unidad de Cuidados Intensivos (UCI). Durante su estadía en UCI, el paciente cursó con una neumonía asociada a ventilador mecánico por Pseudomonas aeruginosa y una bacteriemia por Acinetobacter baumannii multiresistentes. La eliminación de gusanos adultos por la cavidad oral continuó hasta varios días posteriores a la última cirugía.

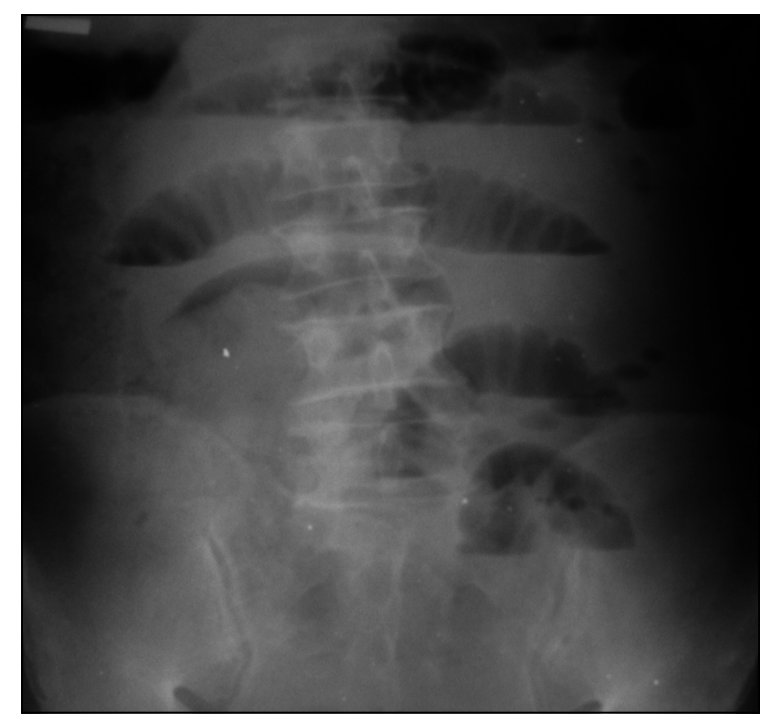

Figura 1. Radiografía abdomen simple de pie. Evidencia de dilatación de asas intestinales y niveles hidroaéreos.

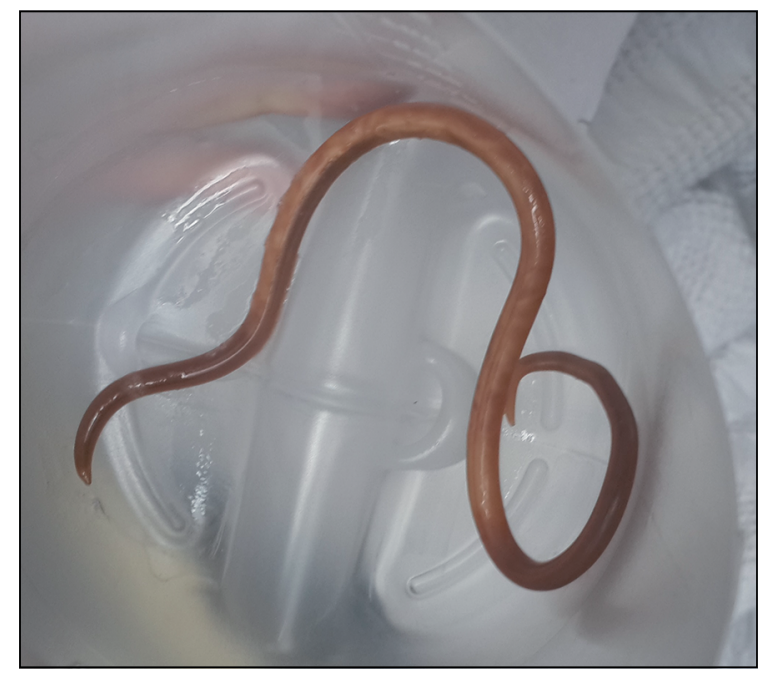

Figura 2. Se observa ejemplar adulto de Ascaris lumbricoides extraído por laparotomía exploratoria.

\section{Discusión}

La mayor incidencia de ascariasis se describe entre los dos y 10 años de edad, declinando significativamente a partir de los 15 años. Del mismo modo, las complicaciones debidas a este parásito, también predominan en la infancia, debido al pequeño tamaño de sus órganos y a las dimensiones que puede alcanzar un ejemplar adulto ${ }^{12,13}$.

Describimos el caso clínico de una obstrucción intestinal por múltiples ovillos de $A$. lumbricoides en una persona mayor de 80 años, condición no sólo infrecuente en personas adultas, siendo excepcional en adultos mayores.

La obstrucción intestinal o biliar, colangio-hepatitis, absceso hepático, pancreatitis, apendicitis aguda y perforación intestinal son complicaciones que se producen 
debido al gran número de ejemplares y a los movimientos de migración intraluminal que presentan.

En un inicio no se consideró dentro del diagnóstico diferencial, la obstrucción por ovillos de $A$. lumbricoides, probablemente debido a que la atención se realizó en un hospital ubicado en una zona no endémica. Sin embargo, si analizamos retrospectivamente los datos, como la procedencia de la Amazonía peruana, región de alta endemicidad, la ausencia de patologías previas, la presentación subaguda del cuadro clínico y la inespecificidad de los análisis de laboratorio; entonces dicho diagnóstico pudo ser considerado.

En este paciente, el diagnóstico de obstrucción intestinal se realizó en base a las manifestaciones clínicas y a los hallazgos en la radiografía de abdomen simple de pie. La avanzada edad y la ausencia de antecedentes quirúrgicos, hizo considerar la posibilidad de obstrucción mecánica por alguna neoplasia; sin embargo, contrarrestaba la presentación brusca de los síntomas.

El estudio con ecografía abdominal se recomienda cuando existe la sospecha de invasión en la vía hepatobiliar; o la búsqueda de parásitos en las deposiciones, que en circunstancias semejantes, han evitado la cirugía en adultos mayores, con buenos resultados ${ }^{14,15}$.

El manejo de la obstrucción intestinal por A. lumbricoides es médico y la conducta quirúrgica debe ser expectante, debido a la elevada morbilidad intra y postoperatoria, tal como ocurrió en nuestro paciente, quien además de requerir manejo y monitoreo del estado crítico en la UCI, presentó infecciones intrahospitalarias graves ${ }^{14}$.

Si el manejo médico fracasa, la cirugía de urgencia es la principal indicación. Según Sönmez y Hershkovitz, la técnica quirúrgica dependerá de los hallazgos intraoperatorios, describiendo la realización de diversos procedimientos desde la resección intestinal, en el caso que exista evidencia de gangrena o infarto intestinal, hasta la enterostomía, cuando se encuentre perforación, o bien una enterotomía si el intestino está indemne y sólo se busca la extracción de los gusanos. Siempre se debe tener la precaución de no liberar los ejemplares adultos o huevos en la cavidad abdominal ${ }^{14,16}$.

En el caso de nuestro paciente, no obstante, el diagnóstico fue en el intraoperatorio, la avanzada edad del paciente hizo optar por no prolongar el acto quirúrgico y elegir el manejo médico posterior. Lamentablemente, la evolución desfavorable conllevó a una segunda cirugía de urgencia para resolver el problema. Es discutible, si la primera intervención debió ser la definitiva.

Se debe tener en consideración, la actual disponibilidad de la endoscopia como posible herramienta terapéutica. Pekery cols., comunicaron el caso de un paciente de 78 años, con obstrucción intestinal por A. lumbricoides, en quien se lograron extraer múltiples gusanos adultos mediante esta técnica, siendo tratado posteriormente con mebendazol, con buen resultado ${ }^{17}$.

Finalmente, consideramos que el paciente tuvo una presunta obstrucción biliar temporal por $A$. lumbricoides, debido a la presencia de ictericia, hiperbilirrubinemia directa y discreta elevación de la GGT; hallazgos que pudieron direccionar a la exploración de la vía biliar durante el acto quirúrgico.

\section{Conclusión}

Los ovillos de ejemplares de $A$. lumbricoides debe considerarse siempre entre los diagnósticos diferenciales de obstrucción intestinal, en pacientes procedentes de áreas endémicas de esta parasitosis, que acuden a las salas de urgencias, indistintamente de la edad. El manejo debe ser médico y quirúrgico expectante.

\section{Resumen}

Ascaris lumbricoides es considerado el nemátodo intestinal de mayor tamaño. Se presenta con mayor frecuencia en la infancia, representando un verdadero problema médico y de salud pública, especialmente en países en vías de desarrollo. Presentamos el caso de un varón de 83 años, natural y procedente de la región amazónica del Perú, sin antecedentes médicos de relevancia, que ingresa a Servicio de urgencias de nuestro hospital por un cuadro de obstrucción intestinal y presunta obstrucción biliar, requiriendo una intervención quirúrgica inmediata. El diagnóstico final de obstrucción intestinal por la presencia de múltiples ovillos de Ascaris lumbricoides, no fue considerado dentro de los diagnósticos diferenciales previo al acto quirúrgico.

\section{Referencias bibliográficas}

1.- Baba A A, Shera A H, Bhat M A, Hakim S, Sheikh K A, Shah O J. Management of biliary ascariasis in children living in an endemic area. Eur J Pediatr Surg 2010; 20: 187-90.

2.- Mishra P K, Agrawal A, Joshi M, Sanghvi B,
Shah H, Parelkar V. Intestinal obstruction in children due to ascariasis: A tertiary health centre experience. Afr J Paediatr Surg 2008; 5: 65-70.

3.- Marcos L, Maco V, Terashima A, Samalvides F, Gotuzzo E. Prevalencia de parasitosis intestinal en niños del valle del Mantaro, Jauja, Perú. Rev Med Hered 2002; 13: 85-9.
4.- Villamizar E, Méndez M, Bonilla E, Varón H, de Onatra S. Ascaris lumbricoides infestation as a cause of intestinal obstruction in children: experience with 87 cases. J Pediatr Surg 1996; 31: 201-4.

5.- Ibáñez N, Jara C, Guerra A, Díaz E. Prevalencia del enteroparasitismo en escolares de comunidades nativas del Alto Marañón, 
Amazonas, Perú. Rev Peru Med Exp Salud Pública 2004; 21: 126-33.

6.- Chammartin F, Scholte R G, Guimaraes L H, Tanner M, Utzinger J, Vounatsou P. Soil-transmitted helminth infection in South America: a systematic review and geostatistical meta-analysis. Lancet Infect Dis 2013; 13 : 507-18.

7.- Álvarez R, Gutiérrez S, Vargas M, Quero A, Bulnes D, Hernández J. Diferencias clínicas entre oclusión y suboclusión intestinal por Ascaris lumbricoides. Datos que orientan al tratamiento quirúrgico. Acta Pediatr Mex 2011; 32: 156-62.

8.- Botero D, Restrepo M. Capítulo 4. Parasitosis Humanas. Editor Corporación para investigaciones biológicas. Cuarta edición.
2003. Editorial CIB. Medellín: 93-141.

9.- Roy S, Karmacharya P, Raj Aryal M. Diagnosis of intestinal ascariasis in modern era. BMJ Case Rep 2014. doi:10.1136/bcr-2013-200529.

10.- Ospina J, Posada M E. Endoscopy treatment of multiple ascariasis biliary in children. Cases report and review. Rev Col Gastroenterol 2007; 22: 149-54.

11.- Iannacone J, Benites M, Chirinos L. Prevalencia de infección por parásitos intestinales en escolares de primaria de Santiago de Surco, Lima, Perú. Parasitol Latinoam 2006; 61: 54-62.

12.- Haswell-Elkins M, Elkins D, Anderson R $M$. The influence of individual, social group and household factors on the distribution of Ascaris lumbricoides within a community and implications for control strategies. Parasitology
1989; 98: 125-34.

13.- Chungara J, Arévalo R. Ascariosis vía biliar intrahepática: informe de caso. Rev Méd La Paz 2011; 17: 39-45.

14.- Sönmez Tamer G, Tamer Y. Case report: an intestinal obstruction due to ascariasis. Turkiye Parazitol Derg 2009; 33: 185-7.

15.- Sardessai S, Sharma M, Barreto G. Isolated pancreatic duct ascariasis in a child. Indian J Radiol Imaging 2006; 16: 571-2.

16.- Hershkovitz D, Wasserberg N. Large bowel obstruction due to Ascaris lumbricoides. Isr Med Assoc J 2004; 6: 115-6.

17.- Peker K, Kılıç K. Endoscopic diagnosis in Ascaris lumbricoides case with pyloric obstruction. Turkiye Parazitol Derg 2011; 35: 210-3. 